\title{
AVALIAÇÃO DE ESTÍMULOS EMOCIONAIS E REATIVIDADE PSICOFISIOLÓGICA EM PACIENTES COM LESÃO ENCEFÁLICA ADQUIRIDA
}

\section{EVALUATION OF EMOTIONAL STIMULI AND PSYCHOPHYSIOLOGICAL REACTIVITY IN PATIENTS WITH ACQUIRED BRAIN INJURY}

\author{
Fábio Silva1, Isabel Almeida², Sandra Guerreiro², Luís Monteiro ${ }^{1,3,4}$
}

\section{RESUMO}

Introdução: Para o estudo das emoções têm-se utilizado técnicas de autorrelato, nomeadamente, as escalas de valência e de arousal do Self-Assessment Manikin (SAM), que proporcionam uma apreciação cognitiva subjetiva das diferentes dimensões emocionais. No entanto, é legítimo equacionar que esta capacidade de avaliação cognitiva possa estar alterada em pacientes com lesão encefálica adquirida (LEA). Consequentemente, pode haver incongruência na avaliação das suas respostas emocionais. Assim, a avaliação deve incluir outras técnicas complementares, como são as medidas fisiológicas periféricas empiricamente validadas para o estudo das emoções.

Métodos: Avaliamos 36 pacientes com LEA em referência a 33 participantes saudáveis. Ambos os grupos observaram imagens agradáveis, desagradáveis e neutras selecionadas do International Affective Picture System (IAPS), que tinham de classificar através das escalas de valência e de arousal do SAM, enquanto eram registadas as suas respostas fisiológicas periféricas: condutância elétrica da pele (CEP) e ritmo cardíaco (RC).

Resultados: Nas técnicas de autorrelato, os pacientes com LEA fazem uma avaliação da valência diferente, independentemente dos estímulos, em relação aos controles. Já quando consideramos a escala de arousal os pacientes sentem-se mais ativados do que os controles, exceto nos estímulos desagradáveis. Contudo, os resultados obtidos na medição objetiva dos seus correlatos fisiológicos não são congruentes com a avaliação cognitiva que realizam, uma vez que mostraram menor reatividade aos estímulos independentemente da sua condição emocional.

Conclusão: Estes resultados mostram que indivíduos com LEA têm dificuldade em fazer uma avaliação coerente do seu estado de ativação fisiológico. Por essa razão, é altamente recomendado o uso simultâneo de medidas psicofisiológicas.

Palavras-chave: Emoção; lesão encefálica adquirida; medidas de autorrelato; medidas fisiológicas

\section{ABSTRACT}

Introduction: Self-report measures have been used in the study of emotions, namely the valence and arousal scales of the Self-Assessment Manikin (SAM), which provide a subjective cognitive appraisal of different emotional dimensions. However, cognitive assessment ability in patients with acquired brain injury $(\mathrm{ABI})$ may be compromised. Consequently, their emotional responses measured by self-report may be inconsistent. In these cases, the assessment should include complementary techniques, such as peripheral physiological measures empirically validated for the study of emotions.

Method: We evaluated 36 patients with $A B I$ and 33 healthy controls. Both groups watched pleasant, unpleasant and neutral images from the International Affective
Clin Biomed Res. ano;39(4):307-315

1 Instituto de Investigação e Formação Avançada em Ciências e Tecnologias da Saúde (linfacts). Gandra, PRD, Portugal.

2 Centro de Reabilitação Profissional de Gaia (CRPG). Arcozelo, Portugal.

3 Instituto Universitário de Ciências da Saúde (IUCS), Cooperativa de Ensino Superior Politécnico e Universitário (Cespu). Gandra, PRD, Portugal.

4 Neuronal Degeneration \& Regeneration (NeuroGen), Centro de Investigação em Tecnologias e Serviços de Saúde (Cintesis). Porto, Portugal.

Autor correspondente: Luís Monteiro luismc.monteiro@iucs.cespu.pt Instituto Universitário de Ciências da Saúde (IUCS), Cooperativa de Ensino Superior Politécnico e Universitário (Cespu) R. Central de Gandra, 1317. 4585-116, Gandra, PRD, Portugal. 
Silva et al.

Picture System (IAPS) and rated them using SAM valence and arousal scales, while their peripheral physiological responses, consisting of skin conductance response (SCR) and heart rate (HR), were recorded.

Results: In self-report measures, patients with $A B I$ evaluated valence differently, regardless of stimuli, compared to controls. Regarding the arousal scale, patients with $A B I$ reported feeling more aroused when compared to controls, except in unpleasant stimuli. However, the results obtained in the physiological assessment are not consistent with those of the cognitive assessment, as they showed lower reactivity to stimuli regardless of their emotional condition.

Conclusion: These results show that patients with $\mathrm{ABI}$ have more difficulty in making a coherent assessment of their physiological arousal. For this reason, the simultaneous use of psychophysiological measures is highly recommended.

Keywords: Emotion; acquired brain injury; self-report measures; physiological measures

As LEA podem ser definidas como danos cerebrais não relacionados com defeitos congénitos ou doenças degenerativas, que podem causar disfunções temporárias ou permanentes, resultando em incapacidade funcional e em dificuldades de ajustamento psicossocial ${ }^{1}$. Indivíduos com LEA podem apresentar uma diversidade de déficitis de acordo com a natureza e localização da lesão ${ }^{2}$. É frequente encontrar nestes indivíduos alterações significativas nos domínios cognitivo, funcional, emocional, motor, psicossocial, vocacional e familiar, que podem persistir durante décadas ${ }^{3}$.

Ao contrário das alterações de motricidade, que são evidentes em alguns doentes com LEA, as alterações cognitivas, comportamentais e emocionais não se revelam da mesma forma e são menos visíveis inicialmente. Não obstante, são igualmente incapacitantes, sendo mesmo a principal razão pela qual estes indivíduos apresentam dificuldades no ajustamento vocacional, pessoal e social ${ }^{4}$. Embora a literatura tenha correlacionado os déficitis cognitivos e comportamentais com as dificuldades no funcionamento psicossocial ${ }^{5}$, não devemos esquecer que as alterações emocionais constituem outro fator determinante neste domínio ${ }^{6}$.

É sabido que as emoções desempenham um papel extremamente útil e ajudam a direcionar a atenção para estímulos significativos, ajudam no input sensorial, ajustam as tomadas de decisão, preparam respostas comportamentais, facilitam as nossas interações sociais e melhoram a memória episódica ${ }^{7}$. Apesar da emoção humana e todos os mecanismos relacionados com a sua formação ou expressão terem interesse milenar, o seu estudo sob a perspetiva psicobiológica é bastante recente, tendo-se assistido na última década a um grande desenvolvimento das designadas neurociências afetivas ${ }^{8}$. Apesar da compreensão da emoção ser de tanto interesse, acaba por ser surpreendente que o conhecimento acerca deste tema revele um desenvolvimento mais lento comparativamente a outros conceitos, como a memória ou a atenção. A explicação para tal acontecimento parece dever-se ao fato de não haver um consenso quanto à definição de emoção, levando a que, por vezes, não exista um progresso na compreensão científica de emoção ${ }^{9}$.

Lang $^{10}$ refere que a natureza da emoção não pode ser entendida como um simples fenómeno interno e unitário. Pelo contrário, define as emoções como sendo predisposições para a ação, resultantes da ativação de determinados circuitos cerebrais perante a existência de estímulos significativos para o organismo, e que se manifestam através de três sistemas de resposta relativamente independentes: (1) sistema cognitivo ou experiencial subjetivo - é o componente vivencial da experiência emocional; (2) sistema fisiológico - são todas as alterações que acontecem nos sistemas viscerais; e (3) sistema comportamental expressivo - são as alterações na atividade motora e na expressão corporal ${ }^{11,12}$, sendo esta a principal abordagem dos modelos atuais ${ }^{13}$.

Deste modo, estes três componentes não podem, em momento algum, ser considerados de forma isolada ou, nesse caso, estamos perante uma abordagem parcial e incompleta do que é o fenômeno emocional ${ }^{14}$. Ou seja, é recomendado que se faça uma avaliação de todas as suas manifestações: (1) autorrelatos para aceder ao conteúdo experiencial; (2) observação do comportamento externo; e (3) captação das respostas fisiológicas ${ }^{15}$.

Também o importante avanço no uso de técnicas de neuroimagem funcional veio dar uma grande ajuda na compreensão dos processos emocionais ${ }^{8}$. Inicialmente, a emoção era muito associada às estruturas cerebrais envolvidas no sistema límbico, com maior foco na amígdala e no hipotálamo ${ }^{16}$, todavia, há já algum tempo que esta ideia foi questionada ${ }^{17}$. Hoje, as estruturas cerebrais fundamentais para a emoção são razoavelmente conhecidas ${ }^{18}$. A amígdala, a ínsula, o estriado ventral, regiões ventrais do 
giro cingulado anterior e o córtex pré-frontal são, segundo modelos neuropsicológicos da emoção, estruturas pertencentes a um sistema neuronal que permite não só a identificação de estímulos emocionalmente significativos, mas também a produção de respostas afetivas e autonômicas a esses mesmos estímulos ${ }^{19}$. Perante estímulos visuais emocionalmente significativos, há uma maior ativação do córtex visual primário e da amígdala, comparativamente com os neutros, enquanto que, quando se tratam de memórias ou imagens de eventos com conteúdo emocional há uma ativação da ínsula e do cingulado anterior ${ }^{20}$. Estudos de neuroimagem funcional, realizados com indivíduos saudáveis, revelam que existe uma diferenciação hemisférica no processamento de emoções ${ }^{21}$. $\mathrm{Na}$ verdade, o hemisfério direito tem sido considerado pela literatura especializada como sendo o dominante para o processamento emocional ${ }^{22}$, e em especial quando se tratam de emoções negativas ${ }^{23}$. Estímulos desagradáveis provocam ativação da amígdala em ambos os hemisférios, do hipocampo direito e da região occipital medial. Estímulos agradáveis, por sua vez, provocam ativação significativa nas regiões occipitais do hemisfério esquerdo e em determinadas zonas do lobo temporal medial. Perante estímulos com arousal idêntico, a ativação da amígdala é maior quando o estímulo é desagradável, e quanto maior for o arousal destas imagens desagradáveis, maior ativação acontece na amígdala direita e no núcleo caudado esquerdo. Relativamente à valência dos estímulos emocionais, quando os estímulos são agradáveis desencadeia-se a ativação da cabeça do núcleo caudado direito, com extensão ao núcleo acumbens e ao córtex pré-frontal dorsolateral esquerdo ${ }^{24}$.

Alguns modelos da teoria dos processamentos duplos têm sugerido a existência de dois mecanismos de processamento distintos ${ }^{25}$. Por um lado, um mecanismo controlado por processos fundamentalmente subcorticais, que processa a informação de uma forma rápida e muito primitiva e independente da memória operativa. É um mecanismo inconsciente, automático, reflexo e apresenta uma relação direta com a situação estímulo. Por outro lado, um mecanismo que envolve as estruturas corticais e assenta em processos mentais conscientes. Neste caso, o processamento da informação é mais sofisticado, todavia mais lento ${ }^{26}$.

Tudo o que temos vindo a dizer até agora diz respeito a estudos realizados com participantes saudáveis e que não apresentam qualquer tipo de lesão encefálica. Tudo se complica quando se pretende estudar pacientes com LEA.

Como referimos anteriormente, dependendo da natureza e localização das lesões, tanto podem surgir alterações nos domínios cognitivo, funcional, emocional, motor, psicossocial, vocacional, como familiar. A gravidade dos défices reflete-se em limitações funcionais, nas atividades da vida diária, na interação social e familiar, entre tantas outras.

$\mathrm{Na}$ verdade, os estudos sobre emoção em pacientes com LEA são escassos, e os poucos que existem dão mais ênfase às dificuldades que estes pacientes manifestam no reconhecimento de expressões faciais emocionais ${ }^{27}$. Relativamente à avaliação de respostas emocionais em pacientes com LEA, os estudos encontrados não são consensuais. Se por um lado alguns estudos revelam que não há diferenças nas pontuações de valência e arousal entre pacientes e controles ${ }^{28}$, outros sugerem que os pacientes classificam os estímulos agradáveis e desagradáveis como sendo mais ativadores do que os neutros, mas atribuem maior arousal comparativamente com os controles ${ }^{29}$.

Assim, alguns estudos têm apontado para a necessidade de ser feita uma avaliação através de medidas objetivas para minimizar as limitações de uma avaliação subjetiva ${ }^{30}$. Os autorrelatos afetivos podem ser suscetíveis de motivações do sujeito e/ou sofrer distorções subjacentes à lesão $0^{13}$, uma vez que, a capacidade de avaliação cognitiva destes pacientes pode estar alterada e, consequentemente, pode haver incongruência na avaliação das suas respostas emocionais.

Por essa mesma razão, foram medidas as respostas eletrodérmicas e cardiovasculares, as medidas fisiológicas periféricas mais utilizadas para avaliar o sistema nervoso autônomo ${ }^{13}$.

Relativamente à analise do $\mathrm{RC}$ através do número de batimentos por minuto (BPM), a literatura tem referido que os indivíduos com LEA não manifestam diferenças em resposta a estímulos emocionais quando são comparados com os controles ${ }^{28}$. Quanto à CEP, os indivíduos com LEA têm revelado menor reatividade, comparativamente a grupos de controle ${ }^{31,32}$, mas utilizam apenas pacientes com lesões frontais.

Assim, o objetivo primordial deste estudo foi avaliar a interferência que a LEA pode ter em nível das respostas emocionais destes pacientes. Para isso, foram medidas as respostas psicofisiológicas, nomeadamente, as respostas fisiológicas periféricas e as respostas subjetivas a estímulos emocionógenos, selecionados a partir do IAPS ${ }^{33}$. Para a avaliação subjetiva do seu estado foi utilizada a escala de valência e arousal do $\mathrm{SAM}^{34}$, dado tratar-se de um instrumento bastante adequado para a avaliação da componente experiencial da resposta emocional, uma vez que, é livre de influências culturais e não é necessário o uso de linguagem ${ }^{12}$ 
Silva et al.

\section{MÉTODOS}

A amostra é constituída por 69 participantes divididos por dois grupos, o grupo LEA e o grupo de controlo (GC). O grupo LEA é composto por 36 indivíduos com LEA, todos eles recrutados do Centro de Reabilitação Profissional de Gaia (CRPG), onde se encontravam a frequentar um programa de reabilitação neuropsicológica. Destes 36 indivíduos, 20 são do sexo masculino e 16 do sexo feminino, com idades compreendidas entre os 23 e os 62 anos $(M=42.31, D P=10.56)$. A sua escolaridade média é de 9.39 anos $( \pm 2.69)$ e apresentavam tempo pós-lesão médio de 3.00 anos $( \pm 3.58)$. Os indivíduos com LEA apresentavam lesões com diferentes etiologias. 20 tinham como causa da lesão o acidente vascular cerebral (AVC), em 9 dos casos a causa era o traumatismo crânio-encefálico (TCE), em 3 eram encefalites, em 2 era anoxia cerebral, num dos casos era malformação arteriovenosa e por fim, o tumor num indivíduo. O GC é constituído por 33 indivíduos da comunidade em geral e sem LEA, dos quais 20 são do sexo masculino e 13 do sexo feminino, com idades compreendidas entre os 17 e os 61 anos $(M=29.23, D P=15.60)$ e com escolaridade média de 11.55 anos $( \pm 1.31)$.

Para induzir as respostas emocionais foram utilizados estímulos visuais. Foram selecionadas doze imagens (Figura 1) do IAPS, sendo que das doze imagens, quatro eram agradáveis, quatro desagradáveis e quatro neutras. As imagens desagradáveis apresentavam pontuações de valência mais baixas e de arousal mais elevadas (valência $\leq 4$; arousal $\geq 5.5$ ), as imagens agradáveis eram pontuadas com valores de valência e de arousal mais elevados (valência $\geq 7$; arousal $\geq 3$ ) e as neutras apresentavam pontuações de valência e arousal mais baixas (valência $\leq 5.5$; arousal $\leq 3.5$ ).

Recorreu-se ao software Presentation 0.71 (Neurobehavioral Systems, Inc), instalado na unidade de estimulação (computador portátil), para apresentar os estímulos que iam surgindo de forma aleatória ao longo da apresentação.

Para recolher os autorrelatos de valência e arousal de cada um dos estímulos, utilizaram-se as escalas pictográficas de valência e intensidade do SAM. A escala SAM para a valência varia entre valores de 1 e 9 , sendo que, a cada um dos números corresponde uma figura humana com uma expressão facial que varia desde uma aparência de desagradado a agradado. A escala SAM para o arousal varia também ela de valores de 1 a 9 e a cada um dos números corresponde uma figura humana com relâmpagos na zona do peito, representando níveis crescentes de ativação fisiológica. Para cada estímulo, o indivíduo assinala uma imagem em cada uma das escalas ${ }^{35}$.
As alterações fisiológicas da emoção foram medidas através da CEP e do RC. Foi utilizado um polígrafo eletrónico da marca BIOPAC (Goleta, USA), modelo MP100, que se encontrava equipado com um amplificador monocanal para registo da CEP, modelo GSR100C (corrente contínua e voltagem constante) e um amplificador de sinal fotopletismográfico, modelo PPG100C, para registo do RC. Para a captação da CEP foram utilizados dois eletrodos $\mathrm{Ag}-\mathrm{Ag} / \mathrm{Cl}$ reutilizáveis, não polarizáveis, modelo TSD203, e para a captação do RC (pulso radial) via alterações da pressão sanguínea foi utilizado um transducer fotoelétrico, modelo TSD100.

Para poder trabalhar com o polígrafo, este encontrava-se instalado num computador onde também estava instalado o software Acqknowledge Versão 3.7.1, da marca BIOPAC, para registo e análise dos dados recolhidos. O polígrafo encontrava-se calibrado segundo as recomendações da marca.

Para a colocação dos eletrodos foi utilizado um gel eletrolítico hipo-saturado e isotônico, com a referência GEL 100.

Antes de dar início ao estudo, este foi aprovado pelo conselho diretivo do CRPG.

Os dados do grupo LEA foram recolhidos no CRPG e os dados do GC foram recolhidos no laboratório de Psicofisiologia do Instituto Universitário de Ciências da Saúde (IUCS). Para evitar interferências de variáveis parasitas à investigação, foram mantidas, em ambos os locais de recolha de dados, as mesmas condições de luz, temperatura, som e disposição dos equipamentos.

Inicialmente, todos os participantes foram informados acerca do objetivo da investigação, sendo recolhido o seu consentimento informado para a coleta de dados, sempre com a garantia de confidencialidade e anonimato.

Em seguida, foi realizada uma minientrevista para coleta dos dados sociodemográficos do participante.

Terminada esta etapa, os participantes eram convidados a sentar-se confortavelmente em frente à unidade de estimulação onde seriam apresentados os estímulos do IAPS. Após este passo, os participantes eram informados de que iriam visualizar imagens e seriam realizados registos psicofisiológicos, motivo pelo qual deveriam ao máximo evitar fazer qualquer movimento.

Foi explicado que, após cada estímulo, teriam de fazer uma avaliação do mesmo através da escala de valência e arousal do SAM.

Terminada a explicação e dadas todas as instruções, era realizado um ensaio prático para garantir a compreensão por parte do participante, procedendo-se, de seguida, à colocação dos sensores para captação das respostas psicofisiológicas.

Para a recolha da CEP realizou-se uma montagem bipolar exossomática estandardizada - dois eletrodos ativos posicionados na face palmar das falanges 
médias da mão dominante, um no indicador e o segundo no dedo médio. Para o RC, foi colocado um transducer pletismográfico fotoelétrico na falange distal do dedo médio da mão não dominante.

Após a montagem e calibragem dos equipamentos, segundo recomendações da marca, iniciou-se o registo dos sinais fisiológicos em repouso com duração de 60 s, correspondendo à linha basal. Passado este tempo, era iniciada a apresentação dos estímulos com a seguinte sequência: (1) ponto de fixação (3s); (2) estímulo (negativo, positivo ou neutro) (10s); (3) instrução para avaliar o estímulo nas escalas de valência e arousal do SAM (3s); (4) escala de valência (5s); (5) escala de arousal (5s); e iniciava novamente com ponto de fixação (3s) até terminar a apresentação dos doze estímulos (Figura 2).

A sequência dos estímulos era aleatória para controlar o efeito de ordem.

A análise estatística foi feita através do software Statistical Package for the Social Sciences (SPSS), versão 25 . Foram utilizadas medidas de tendência central e medidas de dispersão (média e desviopadrão) e distribuição de frequência para os dados sociodemográficos. Foi realizado o teste $T$-Student para amostras independentes para verificar se havia diferenças entre as pontuações dos autorrelatos e as pontuações das medidas fisiológicas periféricas entre o grupo LEA e o GC.

Consideraram-se resultados com $p \leq .05$ significativos. Resultados de $p>.05$ e $\leq .10$ foram considerados marginalmente significativos.

\section{RESULTADOS}

Relativamente à valência (Tabela 1), na condição emocional desagradável, verificamos que o grupo LEA apresenta pontuações de valência $(M=1.92$, $D P=1.25)$ significativamente inferiores $(t=-3.64$, $p \leq .01)$ às pontuações apresentadas pelo $\mathrm{GC}(M=2.46$, $D P=1.24)$. Na condição emocional neutro, o grupo LEA apresenta pontuações de valência $(M=5.51$, $D P=1.30)$ significativamente superiores $(t=2.94$, $p \leq .01)$, comparativamente ao $\mathrm{GC}(M=5.14, D P$ $=.63$ ). Para a condição emocional agradável, à semelhança do que acontece na condição emocional neutro, o grupo LEA apresenta pontuações de valência $(M=7.19, D P=1.45)$ significativamente superiores $(t=2.19, p \leq .05)$, comparativamente ao $\mathrm{GC}(M=6.82, D P=1.35)$.

Em relação ao arousal (Tabela 2), na condição emocional neutro, o grupo LEA revelou pontuações de arousal $(M=3.36, D P=2.15)$ significativamente superiores $(t=5.79, p \leq .01)$, comparativamente às pontuações do $\mathrm{GC}(M=2.05, D P=1.50)$. O mesmo acontece para a condição emocional agradável, o grupo LEA apresenta pontuações de arousal $(M=5.74$, $D P=2.34)$ significativamente superiores $(t=5.08$, $p \leq .01)$, quando comparado com as pontuações obtidas pelo $\mathrm{GC}(M=4.31, D P=2.32)$. Para a condição emocional desagradável, não foram encontradas diferenças estatisticamente significativas entre os dois grupos.

Tabela 1: Média e (desvio-padrão) das pontuações de valência.

\begin{tabular}{|c|c|c|c|c|c|c|c|c|}
\hline \multirow{2}{*}{$\begin{array}{l}\text { Condição } \\
\text { emocional }\end{array}$} & \multirow{2}{*}{$N^{\circ}$ foto } & \multirow{2}{*}{ Estímulo } & \multirow{2}{*}{ Grupo LEA } & \multirow{2}{*}{$\begin{array}{l}\text { Grupo de } \\
\text { controle }\end{array}$} & \multirow{2}{*}{$t$} & \multirow{2}{*}{$p$} & \multicolumn{2}{|c|}{$95 \%$ IC } \\
\hline & & & & & & & $L I$ & $L S$ \\
\hline \multirow{5}{*}{ Desagradável } & 3000 & Mutilação & $1.33(.59)$ & $1.82(.85)$ & -2.79 & $\leq .01$ & -.83 & -.14 \\
\hline & 6560 & Arma & $2.03(1.25)$ & $2.09(.95)$ & -.23 & .82 & -.60 & -.48 \\
\hline & 9901 & Acidente & $1.78(1.15)$ & $2.67(1.24)$ & -3.09 & $\leq .01$ & -1.46 & -.31 \\
\hline & 1120 & Cobra & $2.50(1.54)$ & $3.27(1.35)$ & -2.21 & $\leq .05$ & -1.47 & -.04 \\
\hline & Total & & $1.92(1.25)$ & $2.46(1.24)$ & -3.64 & $\leq .01$ & -.84 & -.25 \\
\hline \multirow{5}{*}{ Neutro } & 7006 & Prato & $5.11(1.39)$ & $5.12(.42)$ & -.04 & .97 & -.51 & .49 \\
\hline & 7010 & Cesto & $5.72(1.23)$ & $5.09(.72)$ & 2.56 & $\leq .01$ & .14 & 1.12 \\
\hline & 7002 & Toalha & $5.33(1.04)$ & $5.09(.68)$ & 1.13 & $\leq .05$ & -.18 & .67 \\
\hline & 7004 & Colher & $5.86(1.40)$ & $5.27(.67)$ & 2.20 & .26 & .05 & 1.12 \\
\hline & Total & & $5.51(1.30)$ & $5.14(.63)$ & 2.94 & $\leq .01$ & .12 & .61 \\
\hline \multirow{5}{*}{ Agradável } & 7400 & Chocolate & $7.31(1.28)$ & $6.88(1.36)$ & 1.34 & .19 & -.21 & 1.06 \\
\hline & 4608 & Erótico & $6.97(1.36)$ & $6.45(1.52)$ & 1.48 & .14 & -.18 & 1.22 \\
\hline & 5621 & Aventura & $6.78(1.82)$ & $6.64(1.32)$ & .37 & .72 & -.63 & .91 \\
\hline & 5760 & Paisagem & $7.72(1.11)$ & $7.30(1.05)$ & 1.61 & .11 & -.10 & .93 \\
\hline & Total & & $7.19(1.45)$ & $6.82(1.35)$ & 2.19 & $\leq .05$ & .38 & .71 \\
\hline
\end{tabular}

Nota: IC = intervalo de confiança; $\mathrm{LI}=$ limite inferior; $\mathrm{LS}$ = limite superior 
Silva et al.

Tabela 2: Média e (desvio-padrão) das pontuações de arousal.

\begin{tabular}{|c|c|c|c|c|c|c|c|c|}
\hline \multirow{2}{*}{$\begin{array}{l}\text { Condição } \\
\text { emocional }\end{array}$} & \multirow{2}{*}{$\begin{array}{c}N^{\circ} \\
\text { foto }\end{array}$} & \multirow{2}{*}{ Estímulo } & \multirow{2}{*}{ Grupo LEA } & \multirow{2}{*}{$\begin{array}{l}\text { Grupo de } \\
\text { controle }\end{array}$} & \multirow{2}{*}{$t$} & \multirow{2}{*}{$p$} & \multicolumn{2}{|c|}{$95 \%$ IC } \\
\hline & & & & & & & $L I$ & $L S$ \\
\hline & 3000 & Mutilação & $6.42(3.04)$ & $6.67(1.65)$ & -.42 & .68 & -1.4 & .94 \\
\hline & 6560 & Arma & $6.69(2.40)$ & $6.58(1.70)$ & .24 & .82 & -.89 & 1.13 \\
\hline \multirow[t]{5}{*}{ Desagradável } & 9901 & Acidente & $6.61(2.61)$ & $5.52(1.87)$ & 1.99 & $\leq .05$ & -.01 & 2.20 \\
\hline & 1120 & Cobra & $5.56(2.72)$ & $5.36(2.03)$ & .33 & .74 & -.97 & 1.35 \\
\hline & Total & & $6.31(2.72)$ & $6.03(1.89)$ & .97 & .33 & -.28 & -.84 \\
\hline & 7006 & Prato & $2.81(1.80)$ & $2.12(1.58)$ & 1.67 & .10 & -.13 & 1.50 \\
\hline & 7010 & Cesto & $3.42(2.14)$ & $1.97(1.63)$ & 3.14 & $\leq .01$ & .53 & 2.37 \\
\hline \multirow[t]{5}{*}{ Neutro } & 7002 & Toalha & $3.44(2.35)$ & $2.21(1.47)$ & 2.58 & $\leq .01$ & .28 & 2.18 \\
\hline & 7004 & Colher & $3.69(2.27)$ & $1.91(1.36)$ & 3.93 & $\leq .01$ & .88 & 2.69 \\
\hline & Total & & $3.36(2.15)$ & $2.05(1.50)$ & 5.79 & $\leq .01$ & .86 & 1.75 \\
\hline & 7400 & Chocolate & $5.33(2.29)$ & $3.91(2.19)$ & 2.64 & $\leq .01$ & .35 & 2.50 \\
\hline & 4608 & Erótico & $5.59(2.41)$ & $4.42(2.25)$ & 2.04 & $\leq .05$ & .03 & 2.30 \\
\hline \multirow[t]{3}{*}{ Agradável } & 5621 & Aventura & $6.33(2.00)$ & $4.76(2.31)$ & 3.04 & $\leq .01$ & .54 & 2.61 \\
\hline & 5760 & Paisagem & $5.72(2.58)$ & $4.15(2.53)$ & 2.55 & $\leq .01$ & .34 & 2.80 \\
\hline & Total & & $5.74(2.34)$ & $4.31(2.32)$ & 5.08 & $\leq .01$ & .88 & 1.99 \\
\hline
\end{tabular}

Nota: IC = intervalo de confiança; LI = limite inferior; LS = limite superior

Relativamente à média das amplitudes de CEP (Tabela 3), verificamos que na condição emocional desagradável, o grupo LEA apresenta pontuações médias de CEP $(M=.178, D P=.116)$ significativamente inferiores $(t=-6.99, p \leq .01)$ às pontuações apresentadas pelo $\mathrm{GC}(M=.298, D P=.167)$. Na condição emocional neutro as pontuações médias de CEP apresentadas pelo grupo LEA $(M=.178, D P=.114)$ são também significativamente inferiores $(t=-7.07, p \leq .01)$, comparativamente às pontuações médias de CEP do GC $(M=.298, D P=.165)$. O mesmo acontece para a condição emocional agradável onde as pontuações médias de CEP do grupo LEA $(M=.177, D P=.115)$ são significativamente inferiores $(t=-7.26, p \leq .01)$, comparativamente ao $\mathrm{GC}(M=.299, D P=.163)$.
Olhando agora para o RC (Tabela 4), medido através dos BPM, relativamente à condição emocional desagradável, o grupo LEA apresenta pontuações de BPM $(M=76.35, D P=10.22)$ marginalmente significativas inferiores $(t=-1.81$, $p=.07)$, comparativamente às pontuações de BPM apresentadas pelo $G C(M=78.89, D P=12.92)$. Para a condição emocional agradável acontece o mesmo, ou seja, o grupo LEA apresenta pontuações de BPM $(M=76.81, D P=10.33)$ marginalmente significativas inferiores $(t=-1.80, p=.07$ ) relativamente às pontuações de BPM apresentadas pelo $\mathrm{GC}(M=79.37, D P=13.16)$. Para a condição emocional neutro não foram encontradas diferenças estatisticamente significativas.

Tabela 3: Média e (desvio-padrão) das amplitudes de CEP.

\begin{tabular}{lcccccc}
\hline $\begin{array}{l}\text { Condição } \\
\text { emocional }\end{array}$ & Grupo LEA & Grupo de controle & $\boldsymbol{t}$ & $\boldsymbol{p}$ & \multicolumn{2}{c}{$\mathbf{9 5 \%}$ IC } \\
\cline { 2 - 7 } Desagradável & $.178(.116)$ & $.298(.167)$ & -6.99 & $\leq .01$ & -.154 & -.087 \\
Neutro & $.178(.114)$ & $.298(.165)$ & -7.07 & $\leq .01$ & -.154 & -.087 \\
Agradável & $.177(.115)$ & $.299(.163)$ & -7.26 & $\leq .01$ & -.156 & -.089 \\
\hline
\end{tabular}

Nota: IC = intervalo de confiança; LI = limite inferior; $L S=$ limite superior

Tabela 4: Média e (desvio-padrão) do RC.

\begin{tabular}{lcccccc}
\hline $\begin{array}{l}\text { Condição } \\
\text { emocional }\end{array}$ & Grupo LEA & Grupo de controle & $\boldsymbol{t}$ & $\boldsymbol{p}$ & \multicolumn{2}{c}{$\mathbf{9 5 \%}$ IC } \\
\hline Desagradável & $76.35(10.22)$ & $78.89(12.92)$ & -1.81 & .07 & -5.29 & .23 \\
Neutro & $76.70(10.28)$ & $78.26(12.32)$ & -1.14 & .26 & -4.25 & 1.13 \\
Agradável & $76.81(10.33)$ & $79.37(13.16)$ & -1.80 & .07 & -5.36 & .24 \\
\hline
\end{tabular}

Nota: IC = intervalo de confiança; LI = limite inferior; LS = limite superior 


\section{DISCUSSÃO}

Como referido anteriormente, este estudo teve como objetivo avaliar a interferência que a LEA pode ter nas respostas emocionais, nomeadamente, nas respostas fisiológicas periféricas e na avaliação subjetiva que os indivíduos fazem de estímulos emocionais.

No que diz respeito às medidas subjetivas, os nossos resultados mostram que, relativamente à valência, os indivíduos com LEA avaliam os estímulos desagradáveis como mais desagradáveis, comparativamente ao GC. Já os estímulos agradáveis e neutros são avaliados como mais positivos, comparativamente aos controles. Estes resultados surgem em oposição ao que vem sendo referido na literatura, onde tem sido referenciado que estes indivíduos fazem avaliações de valência semelhantes às de grupos controles ${ }^{28}$. Porém, os resultados referentes ao arousal mostram que os indivíduos com LEA avaliam os estímulos emocionais agradáveis e neutros como mais ativadores, comparativamente ao GC, sendo que, estes resultados vão de encontro ao que tem sido concluído em alguns estudos ${ }^{29}$. Estes achados vêm dar suporte a estudos que dizem haver áreas cerebrais diretamente relacionadas com a classificação verbal das dimensões de valência e arousa ${ }^{\beta 6}$. Avalência tem sido associada a maior ativação da amígdala e do córtex insular, enquanto que, o arousal tem sido correlacionado com maior ativação talâmica e frontomedial ${ }^{36}$. De certo modo, estes resultados são congruentes com a literatura, que tem referido os indivíduos com LEA como indivíduos com dificuldades em reconhecer expressões faciais emocionais ${ }^{27}$. Desta feita, podemos dizer que a sua capacidade de avaliar imagens emocionalmente significativas aparece comprometida.

Relativamente às medidas fisiológicas periféricas, os participantes do grupo LEA apresentaram menor reatividade de CEP em todas as condições emocionais, comparativamente ao $\mathrm{GC}$, resultados que estão em concordância com estudos anteriores ${ }^{31,32}$. Segundo Damásio e colaboradores ${ }^{37}$, são os indivíduos com lesões frontais, especialmente, indivíduos com lesões no córtex frontal ventromedial que apresentam alterações em nível das respostas autonômicas a estímulos emocionalmente significativos. Assim, a atividade eletrodérmica parece estar diretamente relacionada com o córtex pré-frontal ${ }^{38}$.

Quanto ao RC, foram encontradas diferenças marginalmente significativas entre os grupos, nas condições emocionais desagradável e agradável. Foi possível verificar que o grupo LEA apresenta uma maior desaceleração cardíaca, o que nos leva a crer que esteja diretamente relacionada com o reflexo de orientação, que tem a função de aumentar a sensibilidade sensorial e facilitar a perceção do estímulo e que, de entre várias alterações psicofisiológicas, provoca uma maior desaceleração do $\mathrm{RC}^{39}$. Deste modo, os nossos resultados sugerem que os participantes do grupo LEA direcionaram maior atenção para a visualização dos estímulos aquando da sua apresentação.

Os resultados mostram que estes indivíduos apresentam alterações a nível das respostas emocionais e são, ainda, incapazes de fazer uma avaliação do seu estado fisiológico congruente com a sua reatividade. Alguns estudos ${ }^{36}$ têm especulado que nestes casos os indivíduos fazem uma avaliação não baseada no seu estado interno, mas antes uma avaliação influenciada pelo conhecimento conceptual relacionada com o estímulo emocional apresentado. Quanto à baixa atividade fisiológica periférica, a literatura tem fornecido evidências de que, em indivíduos com LEAé comum encontrar este padrão de resposta a estímulos emocionais ${ }^{40}$. No entanto, os resultados devem ser interpretados com cuidado, visto que, esta realidade é bastante sensível devido à heterogeneidade das lesões ${ }^{41}$.

Uma das limitações deste estudo foi precisamente o fato de não ter sido controlado o tipo de lesão e as áreas cerebrais lesadas em cada um dos participantes, sendo recomendado que em investigações futuras esta variável seja tida em conta para poder ao máximo ser controlada. Além disso, foi possível verificar que um elevado número de participantes do grupo LEA encontrava-se poli-medicado para controlar algumas sintomatologias resultantes da lesão encefálica, e estudos têm fornecido evidências de que alguns tipos de medicamentos interferem com a atividade de estruturas cerebrais envolvidas no processamento emocional ${ }^{42}$. Neste sentido, seria pertinente em estudos futuros avaliar se a medicação neste tipo de população, para além da LEA tem alguma implicação nas suas respostas emocionais.

\section{CONCLUSÃO}

Em conclusão, e como referido anteriormente, as emoções são extremamente úteis e ajudam a direcionar a atenção para estímulos significativos, ajudam no input sensorial, ajustam as tomadas de decisão, preparam respostas comportamentais, facilitam as nossas interações sociais e melhoram a memória episódica. Assim, pode ser concluído que indivíduos com LEA possam apresentar dificuldades nestes domínios, uma vez que, o seu processamento emocional parece comprometido. Ainda, estes indivíduos revelam dificuldades em fazer uma avaliação cognitiva subjetiva dos estímulos emocionais sendo, neste caso, recomendado que se utilizem medidas objetivas, designadamente, medidas psicofisiológicas para se estudar emoções nesta população.

\section{Conflitos de interesse}

Os autores declaram não haver conflitos de interesse. 
Silva et al.

\section{REFERÊNCIAS}

1. The world health report 1996: fighting disease, fostering development. Geneva: World Health Organization; 1996.

2. Turner-Stokes L, Pick A, Nair A, Disler PB, Wade DT. Multi-disciplinary rehabilitation for acquired brain injury in adults of working age (review). Cochrane Database Syst Rev. 2015;(12):CD004170. https://doi. org/10.1002/14651858.CD004170. pub3. PMid:26694853.

3. Lezak MD, Howieson DB, Loring DW. Neuropsychological assessment. New York: Oxford University Press; 2004.

4. Ben-Yishay Y, Rattok J, Lakin P, Piasetsky E, Ross B, Silver S, et al. Neuropsychologic rehabilitation: quest for a holistic approach. Semin Neurol. 1985;5(3):252-9. https://doi. org/10.1055/s-2008-1041522.

5. Lezak M. Living with the characterology altered brain injured patient. J Clin Psychiatry. 1978;39(7):592-8. PMid:681289.

6. Hornak J, Rolls E, Wade D. Face and voice expression identification in patients with emotional and behavioural changes following ventral frontal lobe damage. Neuropsychologia. 1996;34(4):24761. https://doi.org/10.1016/00283932(95)00106-9. PMid:8657356.

7. Gross J. Emotion regulation: conceptual and empirical foundations. In: Gross J, editor. Handbook of emotion regulation. New York: The Guilford Press; 2014. p. 3-20.

8. Armony J, Vuilleumier P. The Cambridge handbook of human affective neuroscience. New York: Cambridge University Press; 2013.

9. Barrett LF. Are emotions natural kinds? Perspect Psychol Sci. 2006;1(1):2858. https://doi.org/10.1111/j.17456916.2006.00003.x. PMid:26151184.

10. Lang PJ. A bio-informational theory of emotional imagery. Psychophysiology. 1979;16(6):495-512. https://doi. org/10.1111/j.1469-8986.1979. tb01511.x.

11. Lang PJ. From emotional imagery to the organization of emotion in memory. In: Birbaumer N, Öhman A, editores. The structure of emotion: psychophysiological, cognitive and clinical aspects. Seattle: Hogrefe \& Huber Publishers; 1993. p. 69-92.

12. Lang PJ. The emotion probe: studies of motivation and attention. $\mathrm{Am}$ Psychol. 1995;50(5):372-85. https:// doi.org/10.1037//0003-066x.50.5.372. PMid:7762889.

13. Cacioppo J, Berntson G, Larsen J, Poehlmann K, Ito T. The psychophysiology of emotion. In: Lewis M, Haviland-Jones J, editores. Handbook of emotions. New York: Guilford Press; 2000. p. 173-91.

14. Lang P. Fear reduction and fear behavior: problems in treating a construct. In: Schlien J, editor. Research in psychotherapy. Washington (DC): American Psychological Association; 1968. p. 90-102.

15. Öhman A, Birbaumer N. The structure of emotion: psychophysiological, cognitive and clinical aspects. Seattle: Hogrefe \& Huber Publishers; 1993.

16. Sánchez-Navarro JP, Román F. Amigdala, corteza prefrontal y especializacion hemisferica en la experiencia y expresion emocional. $A n$ Psicol. 2004;20(2):223-40.

17. LeDoux J. The emotional brain: the mysterious underpinnings of emotional life. New York: Simon \& Schuster; 1996.

18. Dolan RJ. Emotion, cognition and behavior. Science. 2002;298(5596):1191-4. https:// doi.org/10.1126/science.1076358. PMid:12424363.

19. Philips ML, Drevets WC, Rauch SL, Lane R. Neurobiology of emotion perception I: the neural basis of normal emotion perception. Biol Psychiatry. 2003;54(5):504-14. https://doi.org/10.1016/s00063223(03)00168-9. PMid:12946879.

20. Phan KL, Wager T, Taylor SF, Liberzon I. Functional neuroanatomy of emotion: A meta-analysis of emotion activation studies in PET and fMRI. Neurolmage. 2002;16(2):33148. https://doi.org/10.1006/ nimg.2002.1087. PMid:12030820.

21. Adolphs R, Damasio H, Tranel D, Damasio AR. Cortical systems for the recognition of emotion in facial expressions.
J Neurosci. 1996;16(23):7678-

87. https://doi.org/10.1523/ JNEUROSCI.16-23-07678.1996. PMid:8922424.

22. Borod JC. Interhemispheric and intrahemispheric control of emotion: A focus on unilateral brain damage. $J$ Consult Clin Psychol. 1992;60(3):33948. https://doi.org/10.1037//0022006x.60.3.339. PMid:1619088.

23. Davidson RJ, Irwin W. The functional neuroanatomy of emotion and affective style. Trends Cogn Sci. 1999;3(1):11-21. https://doi. org/10.1016/s1364-6613(98)01265-0. PMid:10234222.

24. Gerdes AB, Wieser MJ, Mühlberger A, Weyers P, Alpers GW, Plichta MM, et al. Brain activations to emotional pictures are differentially associated with valence and arousal ratings. Front Hum Neurosci. 2010;4:175. https:// doi.org/10.3389/fnhum.2010.00175. PMid:21088708.

25. Evans J, Frankish K. In two minds: dual processes and beyond. Oxford: Oxford University Press; 2009.

26. LeDoux J. Cognitive-emotional interactions in the brain. Cogn Emot. 1989;3(4):267-89. https://doi. org/10.1080/02699938908412709.

27. Leite M, Guerreiro S, Almeida I, Peixoto B. Gandra-Barta for the assessment of facial emotion recognition in acquired brain injury. Acta Neuropsychol. 2017;15(2):127-41. https://doi. org/10.5604/01.3001.0010.2405.

28. Meadows ME, Kaplan RF. Dissociation of autonomic and subjective responses to emotional slides in right hemisphere damaged patients. Neuropsychologia. 1994;32(7):84756. https://doi.org/10.1016/00283932(94)90022-1. PMid:7936167.

29. Sánchez-Navarro JP, Martínez-Selva $\mathrm{JM}$, Román F. Emotional response in patients with frontal brain damage: effects of affective valence and information content. Behav Neurosci. 2005;119(1):87-97. https://doi. org/10.1037/0735-7044.119.1.87. PMid:15727515.

30. Desmet $P$. Measuring emotion: development and application of an instrument to measure emotional responses to products. In: Blythe MA, Overbeeke K, Monk AF, Wright PC, 
editores. Funology: from usability to enjoyment. Dordrecht: Kluwe Academic Publishers; 2003. p. 111-23.

31. Tranel D, Damasio H. Neuroanatomical correlates of electrodermal skin conductance response. Psychophysiology. 1994;31(5):427-38. https://doi. org/10.1111/j.1469-8986.1994. tb01046.x. PMid:7972597.

32. Zahn TP, Grafman J, Tranel D. Frontal lobe lesions and electrodermal activity: effects of significance. Neuropsychologia. 1999;37(11):122741. https://doi.org/10.1016/s00283932(99)00020-2. PMid:10530723.

33. Lang PJ, Bradley MM, Cuthbert BN. International affective picture system (IAPS): affective ratings of pictures and instruction manual. Gainesville: University of Florida; 2008. Technical Report A-8.

34. Bradley MM, Lang PJ. Measuring emotion: the self-assessment manikin and the semantic differential. $J$ Behav
Ther Exp Psychiatry. 1994;25(1):4959. https://doi.org/10.1016/00057916(94)90063-9. PMid:7962581.

35. Lang PJ, Bradley MM, Cuthbert BN. International affective picture system (IAPS): affective ratings of pictures and instruction manual. Gainesville: University of Florida; 2005. Technical Report A-6.

36. Anders S, Lotze M, Erb M, Grodd W, Birbaumer N. Brain activity underlying emotional valence and arousal: a response-related fMRI study. Hum Brain Mapp. 2004;23(4):200-9. https://doi.org/10.1002/hbm.20048. PMid:15449355.

37. Damasio AR, Tranel D, Damasio $H$. Individuals with sociopathic behavior caused by frontal damage fail to respond autonomically to social stimuli. Behav Brain Res. 1990;41(2):81-94. https://doi. org/10.1016/0166-4328(90)90144-4. PMid:2288668.

38. Hugdahl K. Psychophysiology: the mind-body perspective. Cambridge: Harvard University Press; 1995.
39. Martínez-Selva JM. Una revisión de los componentes del reflejo de orientación. An Psicol. 1984;1:167-80.

40. Hopkins MJ, Dywan J, Segalowitz SJ. Altered electrodermal response to facial expression after closed injury. Brain Inj. 2002;16(3):245-57. https:// doi.org/10.1080/02699050110103346. PMid:11874616.

41. Maas Al, Marmarou A, Murray GD, Teasdale SG, Steyerberg EW. Prognosis and clinical trial design in traumatic brain injury: The impact study. J Neurotrauma. 2007;24(2):2328. https://doi.org/10.1089/ neu.2006.0024. PMid:17375987.

42. Takahashi $\mathrm{H}$, Yahata N, Koeda M, Takano A, Asai K, Suhara T, et al. Effects of dopaminergic and serotonergic manipulation on emotional processing: a pharmacological fMRI study. Neurolmage. 2005;27(4):9911001. https://doi.org/10.1016/j. neuroimage.2005.05.039. PMid:15978846.

Recebido: 16 set, 2019 Aceito: 27 out, 2019 